\title{
A Piezo-Driven Compliant Nanopositioning Stage with Large Stroke for Micro/Nano Manipulation
}

\author{
Xiaobo Zhu, Zhijie Wen and Pinkuan Liu \\ State Key Laboratory of Mechanical System and Vibration, School of Mechanical Engineering, Shanghai JiaoTong University, Shanghai \\ 200240, People's Republic of China
}

\begin{abstract}
This paper presents the design, analysis, and testing of a single-axis compliant nanopositioning stage with large stroke driven by piezoelectric actuator for micro/nano manipulation. In the developed stage, a hybrid displacement amplifier integrating two kinds of flexure-based input/output decoupling modules have been developed to achieve a large stroke and precise positioning. The analytical modelling and finite element analysis are given to validate the mechanical performance and a prototype of the proposed stage is fabricated for performance tests. The experimental results show that the developed stage has the travel stroke of $214 \mu \mathrm{m}$ with a resolution of $8 \mathrm{~nm}$. It is demonstrated that the stage can be adequate for micro/nano manipulation.
\end{abstract}

\section{Introduction}

Recently, compliant nanopositioning stages with a large stroke are frequently required in different fields of micro/nanomanipulation.[1, 2] Meanwhile, piezoelectric (PZT) actuators with nanometer resolution, excellent dynamic characteristics, small size, and high driving force have been usually employed in the nanopositioning stages.[3, 4] However, the drawback of the piezoelectric actuator is its limited travel range which are not suitable for requiring a large workspace.

Consequently, a lever-type flexure-based displacement amplifier is widely applied to amplify the limited stroke of PZT in many compliant nanopositioning stages.[5] While is can easily induce parasitic motion errors.[6] Another type of commonly used amplifier refers to bridge-type amplifier, which has compact size, symmetric structure and sufficient amplification ratio.[4, 7] Unfortunately, the nanopositoning stages based on bridge-type amplifier have relatively poor dynamics. In addition, duo to a lateral displacement at the driving end of the stage increases with the increasing of the input displacement, a decoupling mechanism is needed for protect PZT from the lateral forces and bending torques.[8] Therefore, it is a challenge working to design a compliant nanopisitioning stage possessing both low cross-coupling and large stroke simultaneously.

In this paper, a novel compliant piezo-driven nanopositioning stage with large stroke is proposed. A hybrid amplifier, which is composed of double flexure-based amplifers, is developed to achieve a large stroke. In contrast with the commonly amplifier, the novel amplifier is characterized by compact size with large amplification ratio, high dynamics and low cross-coupling.

Meanwhile, two types of compliant decoupling modules are developed to reduce the input and output couplings.Based on the configration of the stage, the analysis models and experimental tests validate that the developed stage achieved the large stroke with nanoscale resolution, low input coupling and compact size. It is demonstrated that a considerable potentical of the stage for the applications in various micro/nano manipulations.

\section{Mechanical design}

The novel compliant nanopositioning stage is shown in Fig.1. It is composed of a symmetrical flexure-based input decoupling module(ABCD), a hybrid amplifier(EFGH), a flexure-based motion guide module(IJKL), and a PZT actuator. Corner-filleted flexure hinges are used in the stage .The PZT actuator adopted in the developed stage can generate a nominal displacement of up to $38 \mu \mathrm{m}$ at the max mum drive voltage of $100 \mathrm{~V}$, and can provide a maximum driving force of $1000 \mathrm{~N}$.

As shown in Fig.1(b), a hybrid-type amplifier, which is a combination of lever-type and bridge-type amplifier, has a compact size with a large amplification ratio and high dynamics. Meanwhile, duo to the symmetric configuration, the amplifier can eliminate the lateral offset error of the output end and gurantee a precise linear output. A couple of input decoupling modules are both mounted between two sides of the PZT. It can reduce the lateral movement perpendicular to the PZT, and protect the PZT from lateral forces and bending torques. In addition, a motion guide module is integrated with the 
moving platform can further ensure a precise positioning

of the output end.

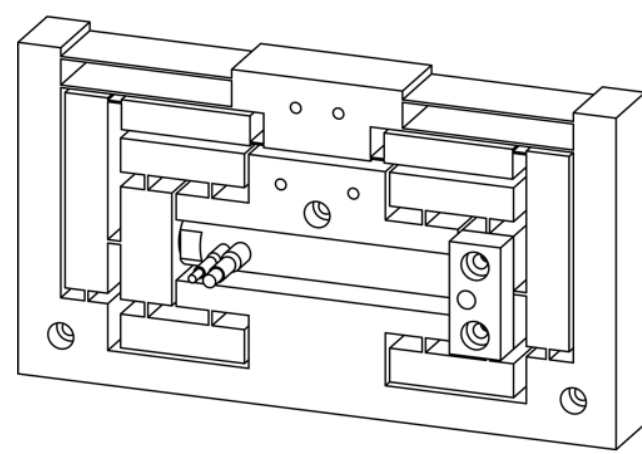

(a)

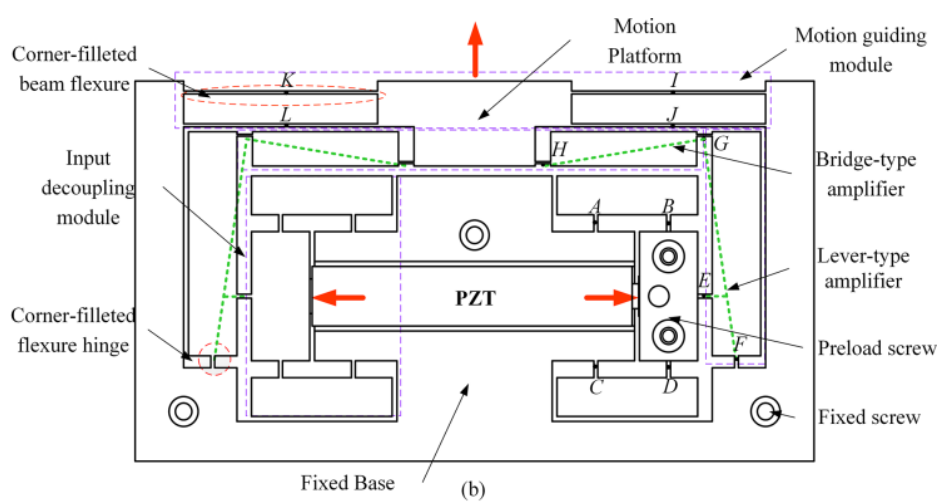

Figure 1. Developed Compliant Stage (a) 3-D Prototype and (b) Schematic Diagram

The motion of the nanopositioning stage is described as follows. When the PZT exerts on the stage, it results in the horizontal displacement of the input decoupling module, which is subsequently amplified by the lever-type and bridge-type amplifiers (EFGH), and in turn, to prompt a precise linear displacement of the moving platform by the motion guide module (IJKL).

\section{Modeling and analysis}

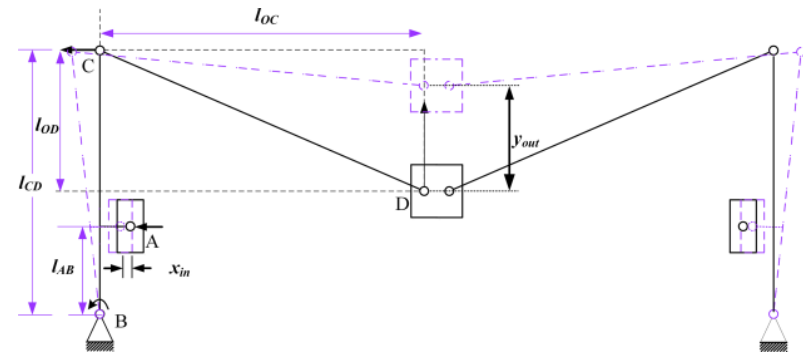

Figure 2. Kinematics of the developed compliant stage

The fundamental structure of the nanopositioning stage is a special five-bar linkage mechanism as illustrated in Fig.2. The input and output displacement are resented by $x_{\text {in }}$ and $y_{\text {out }}$, respectively. The theoretical displacement amplification radio $R_{\text {amp }}$ of the DAM using geometric relations is defined as:

$$
R_{a m p}=\frac{2\left(l_{O D}-\sqrt{l_{O D}{ }^{2}-\left(\frac{l_{B C}}{l_{A B}} x_{i n}+2 l_{O C}\right) \frac{l_{B C}}{l_{A B}} x_{i n}}\right)}{x_{i n}}
$$

According to Eq. (7), it can be seen that the stage has a constant amplification radio, which only depends on the geometric parameters of $l_{A B}, l_{B C}, l_{O C}$ and $l_{O D}$, but has no relations with flexure elements.

The static model of the compliant nanopositioning stage is based on the strain energy method.[9] For
Castigliano's second theorem, the total strain energy of the developed stage is

$$
U=\sum_{i=1}^{10}\left(\int_{0}^{l i} \frac{N_{i}^{2}}{2 E A} d x_{i}\right)+\sum_{i=1}^{10}\left(\int_{0}^{l i} \frac{M_{i}^{2}}{2 E I} d x_{i}\right)+\sum_{i=1}^{10}\left(\int_{0}^{l i} \frac{\alpha S_{i}^{2}}{2 G A} d x_{i}\right)
$$

where $E$ is Young's modulus; $A$ is the cross-section area of the flexure hinges; $I$ is the inertia moment; $G$ is the shear modulus. The normal force $N_{i}$, shearing force $S_{i}$ and bending moment $M_{b i}$ can be expressed by static equilibrium.

For strain energy method, the input displacement $x_{i n}$, output displacement $x_{\text {out }}$, input stiffness of the stage can be obtained as, respectively

$$
\begin{gathered}
x_{\text {in }}=\frac{\partial U}{\partial F_{\text {in }}}=\frac{\delta}{\delta F_{\text {in }}} \mid \sum_{i=1}^{10} \int_{0}^{l_{i}}\left[\frac{N_{i}^{2}}{2 E A}+\frac{M_{b i}^{2}}{2 E I}+\frac{\alpha S_{i}^{2}}{2 G A}\right] d x_{i} \\
x_{\text {out }}=\frac{\partial U^{*}}{\partial F_{11 y}^{*}}=\left.\frac{\partial}{\partial F_{11 y}^{*}}\right|_{F_{11 y}^{*}=0}\left(\sum_{i=8}^{10} \int_{0}^{l_{i}}\left[\frac{N_{i}^{* 2}}{2 E A}+\frac{\alpha S_{i}^{* 2}}{2 G A}+\frac{M_{b i}^{* 2}}{2 E I}\right] d x_{i}\right)
\end{gathered}
$$

$$
k_{i n}=F_{i n} / x_{i n}
$$

It is known that a piezo actuator is compressed when a force is applied. Hence, the stiffness of the PZT and the stage must be taken into account when deducing the maximum output displacement of the stage and can be expressed as

$$
x_{a c t}=\frac{k_{p z t}}{k_{p z t}+k_{i n}} x_{p z t} R_{a m p}
$$

where $k_{p z t}$ is the stiffness of the PZT, $x_{p z t}$ is the maximum nominal displacement of the PZT. 


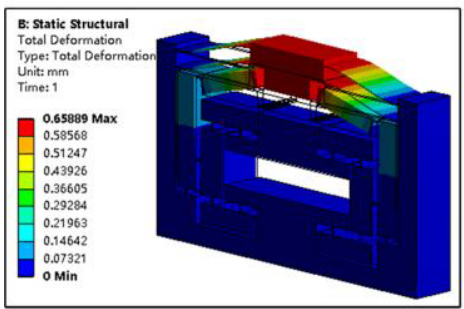

(a)

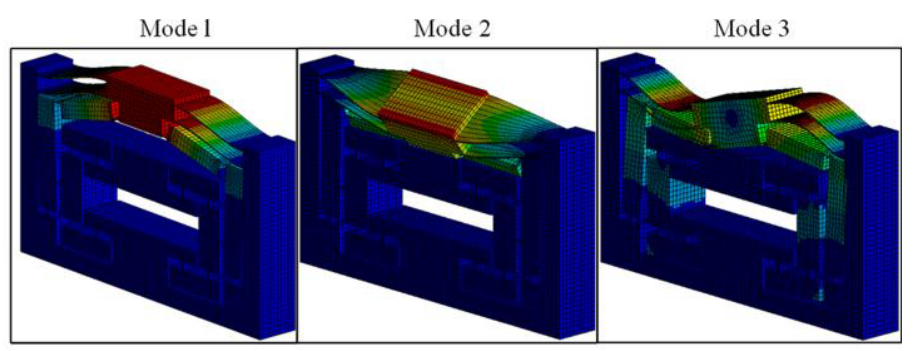

(b)

Figure 3. FEA results of (a) Deformation Behaviour and (b) Mode Shapers from 1st to $3^{\text {rd }}$

Fig. 3 shows the FEA results of the developed stage by commercial software ANSYS. The stage can generate an output displacement of $208 \mu \mathrm{m}$ with the input displacement of $18 \mu \mathrm{m}$ as shown in Fig.3 (a) Thus, it can be deduced that the proposed stage has a displacement amplification ratio of 12.1. And it can be inferred that the input stiffness of the stage is $28.2 \mathrm{~N} / \mu \mathrm{m}$ and the output stiffness of the stage is $2.45 \mathrm{~N} / \mu \mathrm{m}$. In addition, the relevant maximum stress of $52 \mathrm{MPa}$ is much smaller than the yielding stress of the material of aluminum alloy 7075 . Fig.3 (b) demonstrates that the first three natural frequencies of the proposed stage are $202 \mathrm{~Hz}, 1161 \mathrm{~Hz}$ and $1410 \mathrm{~Hz}$, repectively.

\section{Experiment}

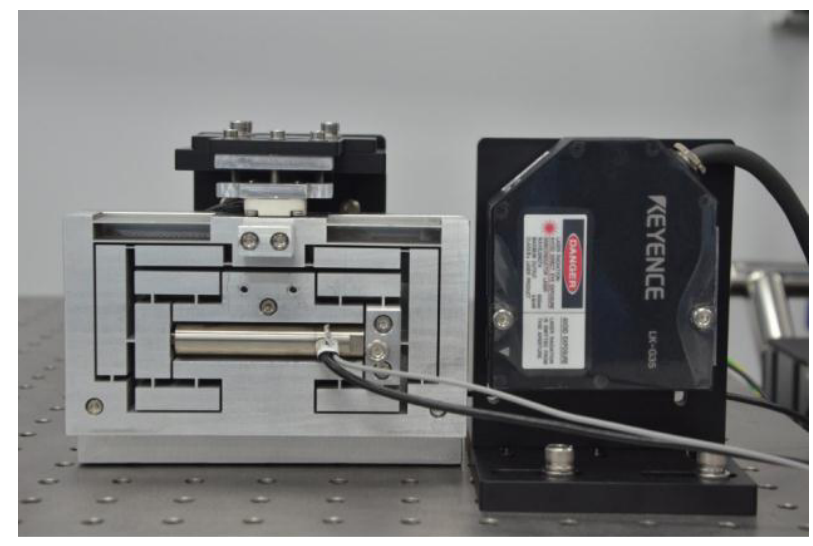

Figure 4. The Manufactured Nanopositioning Stage

Fig. 4 shows that a prototype of the proposed stage with compact size of $92 \times 18 \times 50 \mathrm{~mm}$, which is fabricated using wire electrical discharge machining (WEDM) technique with Aluminum 7075. The positioning stage is driven by a piezo actuator (model: P-841.30, from Physik Instrument, Inc.) with a muti-channel PZT amplifier (model: E-501 from Physik Instrument, Inc.) providing driven voltage $(0-100 \mathrm{~V})$ for the PZT. A capacitive sensor (model: 4810, from MicroSense) is integrated with the stage as a position feedback. A compactRIO 9022 from National Instrument Co. equipped with a 16-bit $\mathrm{ADC} / \mathrm{DAC}$ is applied to carry out a real-time control for the proposed stage.

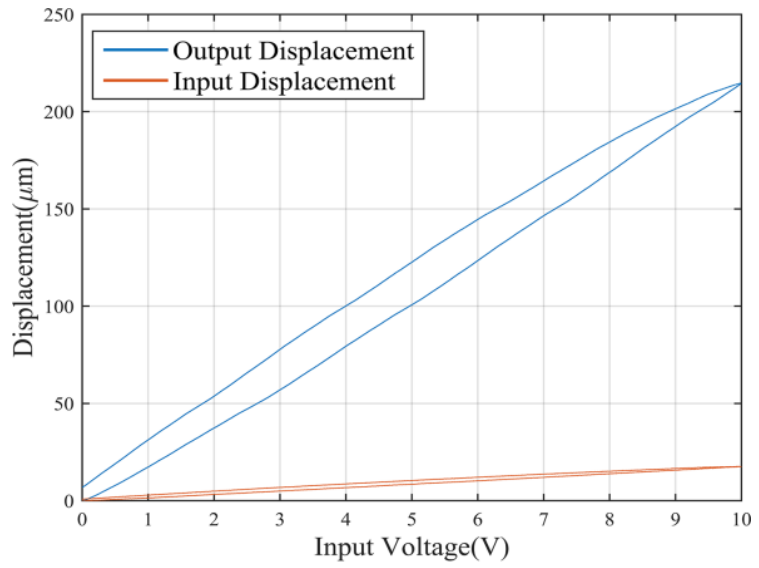

(a)

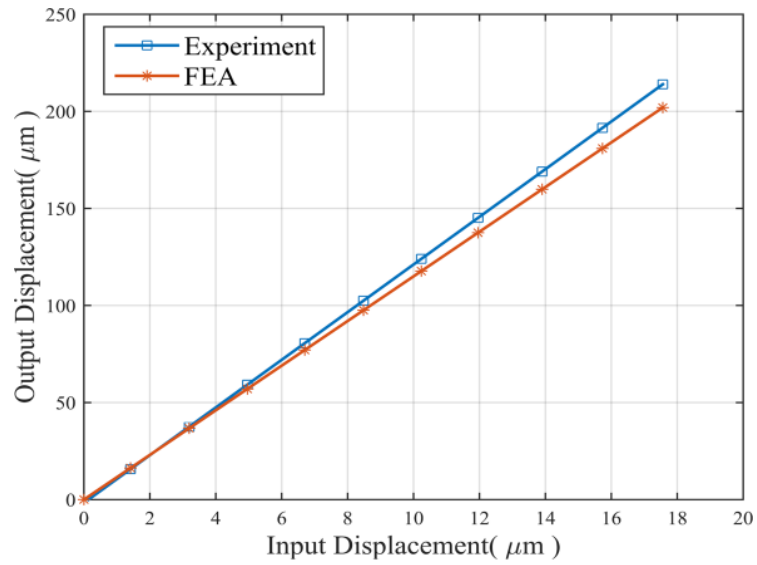

(b)

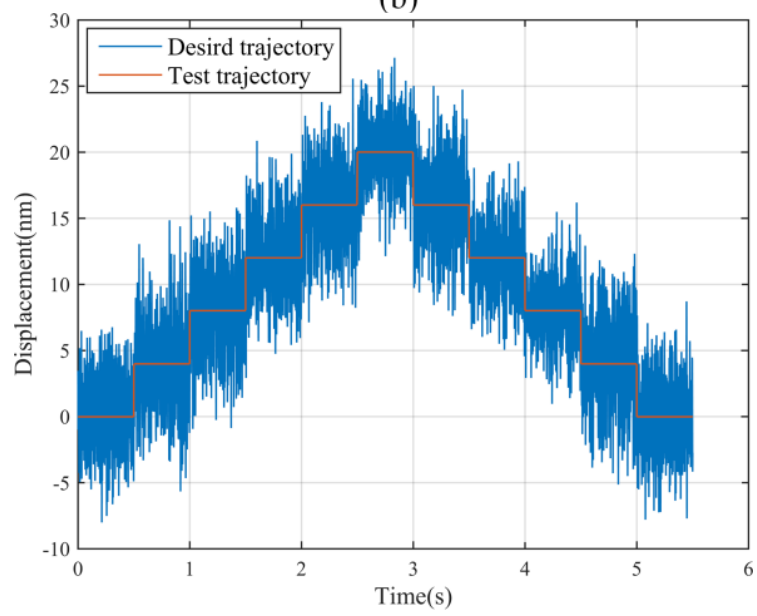

(c) 


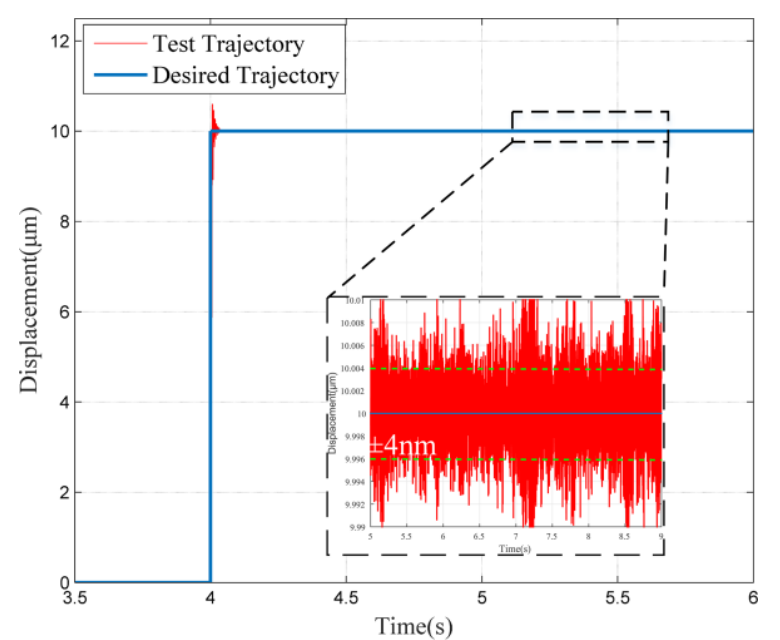

(d)

Figure 5. The Test Results of the Proposed Stage (a)Displacement for Input Voltage from 0 to $100 \mathrm{~V}$, (b) Amplification Ratio, (c) Resolution of $8 \mathrm{~nm}$ and (d) Stepwise Response of $10 \mu \mathrm{m}$

As shown in Fig.5(a), when a $100 \mathrm{~V}$ voltage signal is provided to drive the piezoelectric actuator, the maximum input displacement of the stage is approximately $214 \mu \mathrm{m}$ with respect to the input displacement of $17.7 \mu \mathrm{m}$. The effective amplification ratio of 12.1 is shown in Fig.5(b).

Meanwhile, in close-loop positioning performance test, a minimum resolution of $8 \mathrm{~nm}$ is clearly attained as shown in Fig.5(c). Besides, Fig.5(d) show $\mathrm{s}$ a step response of $10 \mu \mathrm{m}$, which demonstrats that the stage has a rapid transient response (less than $30 \mathrm{~ms}$ ) and steady state error of $\pm 4 \mathrm{~nm}$.

\section{Conclusion}

A pizeo-driven compliant PZT-driven nanopositioning stage with large stroke is developed in this paper. To design an optimized prototype, we establish the kinematic model and perform the FEA validation. Finally, a prototype of the stage is fabricated to carry out the performance test of the stage. The test results show that the proposed stage with a minimum resolution of $8 \mathrm{~nm}$ has a high amplification ratio of 12.1 and a maximum displacement of $214 \mu \mathrm{m}$. It demonstrated that the proposed compact stage is capable of utilizing in the field of micro/nano manipulations.

\section{References}

1. C.-L. Chu, S.-H. Fan, A novel long-travel piezoelectric-driven linear nanopositioning stage, Precision Engineering, 30 (2006) 85-95.

2. P. Gao, S.-M. Swei, Z. Yuan, A new piezodriven precision micropositioning stage utilizing flexure hinges, Nanotechnology, 10 (1999) 394.

3. Y. Yong, S. Moheimani, B.J. Kenton, K. Leang, Invited review article: High-speed flexure-guided nanopositioning: Mechanical design and control issues, Review of scientific instruments, 83 (2012) 121101.

4. J.H. Kim, S.H. Kim, Y.K. Kwak, Development of a piezoelectric actuator using a three-dimensional bridge-type hinge mechanism, Review of scientific instruments, 74 (2003) 2918-2924.

5. S. Choi, S. Han, Y. Han, B. Thompson, A magnification device for precision mechanisms featuring piezoactuators and flexure hinges: Design and experimental validation, Mechanism and Machine Theory, 42 (2007) 1184-1198.

6. J.-J. Kim, Y.-M. Choi, D. Ahn, B. Hwang, D.-G. Gweon, J. Jeong, A millimeter-range flexure-based nano-positioning stage using a self-guided displacement amplification mechanism, Mechanism and Machine Theory, 50 (2012) 109-120.

7. Q. Xu, Y. Li, Analytical modeling, optimization and testing of a compound bridge-type compliant displacement amplifier, Mechanism and machine theory, 46 (2011) 183-200.

8. S. Awtar, A.H. Slocum, Constraint-based design of parallel kinematic XY flexure mechanisms, Journal of Mechanical Design, 129 (2007) 816-830.

9. N. Lobontiu, Compliant mechanisms: design of flexure hinges, CRC press, 2010. 